\title{
Numerical Simulation and Experimental Study on Flow of Polymer Aqueous Solution in Porous Jet Nozzle
}

\author{
Minghui Wei $(\mathbb{D}$, Chenghuai Wu $\mathbb{D}$, and Yanxi Zhou $\mathbb{1}$ \\ College of Mechanical and Electrical Engineering, Southwest Petroleum University, Chengdu, China \\ Correspondence should be addressed to Minghui Wei; wmh881988@163.com
}

Received 30 August 2019; Accepted 9 March 2020; Published 26 March 2020

Academic Editor: Gyorgy Szekely

Copyright ( $\odot 2020$ Minghui Wei et al. This is an open access article distributed under the Creative Commons Attribution License, which permits unrestricted use, distribution, and reproduction in any medium, provided the original work is properly cited.

\begin{abstract}
The addition of a polymer to the jet medium enhances its ability to break rock, and the structure of the nozzle plays a vital role in the full utilization of energy. In this paper, a self-propelled porous jet bit with a support plate is designed, which can prevent the drill bit from jamming due to the jet nozzle against the bottom of the well during the drilling process. And the structural design of the cone-converging nozzle is applied to the forward center nozzle. The polymer additive jet flow field and the pure water jet flow field were compared by numerical simulation and experimental investigation. The results show that the polymer additive jet has a longer isokinetic core, and the rock-breaking volume of the polymer additive jet is much larger than that of the pure water jet, and the optimal spray distance is increased. The forward central jet with the conical convergent nozzle structure has more efficient rock-breaking ability.
\end{abstract}

\section{Introduction}

High-pressure water jet technology is widely used in cutting, crushing, cleaning, and other aspects due to its high material utilization, no thermal effect, concentrated energy, safe operation, and no dust generation. The factors affecting the impact of the water jet are mainly the nozzle structure, the driving force of the jet, the nature of the jet liquid, the spray distance, and so on. The addition of high molecular weight polymer to the jet can effectively reduce the resistance of the fluid, reduce the energy consumption, and improve the isokinetic core of the jet. It was first proposed by Rosler and Bankoff [1]. Li et al. [2] and others added high polymer additives to the jet-breaking rock for experimental research. Studies have shown that, under the same conditions, the rock-breaking volume of the dilute solution jet of polyacrylamide (PAM) is about twice the volume of the pure water jet-breaking rock, and the jetting distance of the largest rock-breaking efficiency is larger than that of the pure water jet. Liu and Li [3,4] of China University of Petroleum measured the flow field of the pure water jet and different concentration additive (PAM) jets through LDV. The experimental results show that the additive jet is longer than the constant velocity core of the pure water jet, and the constant velocity core is the longest when the additive concentration is in the range of $100-200 \mathrm{mg} / \mathrm{L}$. Wang et al. [5-7] used the test method to study the axial dynamic pressure distribution of the polymer additive jet in the submerged state. The results show that the polymer additive can reduce the energy loss of the fluid in the pipeline and at the nozzle, increase the velocity and dynamic pressure of the jet at the nozzle outlet, and increase the constant velocity core of the circular jet. The results of the rock-breaking test show that the polymer additive can improve the rockbreaking ability of the jet. Yang et al. [8-11] used PIV velocimetry to study the abrasive slurry jet under submerged conditions and systematically measured the relationship between the velocity of the fluid and abrasive and the concentration of PAM. It is found that, as the concentration of the polymer in the drilling fluid increases, the velocity of the fluid in the jet and the velocity of the abrasive particles increase correspondingly, and as the concentration of the polymer increases, the convergence of the jet increases. Polyacrylamide has the effect of suppressing the lateral expansion of the jet and reducing the axial attenuation of the jet. However, there are few simulation analyses of the 
additive jet flow field at present, and most of the research studies tend to be experimental research on the effects of actual cutting and crushing [12-19]. Therefore, it is important to strengthen the flow field study of polymer additive jets.

Radial horizontal well technology began in the 1980s. In the past 20 years, radial horizontal well technology has developed rapidly and has the potential to develop unconventional oil and gas [20-24]. This technology requires the use of the high-efficiency rock-breaking water jet bit, high-pressure fluid through small diameter coiled tubing, and high-pressure hose into the jet bit in order to achieve rock-breaking drilling effect. In this case, jet bit is an indispensable technology, which not only needs to break rocks and enlarge holes but also needs to provide traction for the high-pressure hose [25-28]. However, in the actual drilling process, due to the complicated conditions at the bottom of the well, the forward nozzle of the drill bit often contacts with the hard rock layer to be drilled directly. In addition, the forward thrust applied to the nozzle after drilling leads to the forward nozzle being firmly blocked by the rock layer, which is the phenomenon of "stuck" of the water jet bit.

In order to solve the above problems, this paper designs a self-entering multihole jet bit with the support plate. At the same time, the structural design of the conical convergent nozzle is applied to the forward center hole to increase the equal velocity core length to improve the rock-breaking efficiency [29-32]. The flow field of the jet bit is analyzed by the numerical simulation method, and the influence of the support plate on the drilling of the water jet bit and the influence of diffuse flow chip removal are studied. In order to protect oil and gas reservoirs and effectively carry rocks, it is better to add a certain amount of polymer additives in the jet medium, and the jet effect will be different from that of the pure water jet. Through the numerical simulation analysis of the polymer additive jet flow field and pure water jet flow field, the influence of polymer additive on jet rock-breaking effect is explored. The research results can guide the hydraulic parameter design of horizontal drilling.

\section{Nozzle Structure}

As shown in Figure 1, the most important feature of the selfpropelled porous jet bit with the support plate is that the front part of the bit has the support plate, which is used to maintain a certain distance between the bit and the drilling bottom to prevent the water jet bit from jamming during drilling. The forward nozzle is distributed in a structure of $1+3$, that is, one central nozzle and three nozzles distributed in the same circle around the central hole at the same angle. And the fluid ejected will eventually form the perforation with area connectivity within a certain diameter range. Moreover, the structural design of the conical convergent nozzle is applied to the central nozzle, which can minimize the diffusion angle of jet flows, and the higher the density of jet flows, the more concentrated the energy [33]. The structural parameters of the bit mainly include the length of the bit $L_{1}=40 \mathrm{~mm}$, the outer diameter $D_{1}=18 \mathrm{~mm}$, the inner diameter $D_{2}=10 \mathrm{~mm}$, the support plate length $L_{2}=10 \mathrm{~mm}$, the diameter of the forward circular and backward holes $D_{1}=1 \mathrm{~mm}$, the back hole diffusion angle $\alpha_{2}=30^{\circ}$, the diameter of the front central hole $D_{2}=1 \mathrm{~mm}$, the forward hole diffusion angle $\alpha_{3}=15^{\circ}$, and the front central hole cone $\alpha_{1}=13.5^{\circ}$.

Figure 2 is the geometric model of a self-entering porous jet bit with the support plate established by SoildWorks, 3D modeling software. In this paper, a comparative analysis of the polymer additive jet flow field and pure water jet flow field will be built on this model.

\section{Numerical Simulation Model and Boundary Conditions}

3.1. ControlEquation. The research object in this paper is the flow field under the nonsubmerged jet, and there is no violent vortex movement due to the high flow velocity of the polymer additive generated by the self-entering porous jet bit and the area of high Reynolds number inside and outside the bit. The standard $k-\varepsilon$ turbulence model is more suitable for complete turbulence. The transport equation for the standard $\mathrm{k}-\varepsilon$ cycle turbulence model is as follows [34]:

$$
\begin{aligned}
\frac{\partial(\rho k)}{\partial t}+\frac{\partial\left(\rho k u_{i}\right)}{\partial x_{i}}= & \frac{\partial}{\partial x_{j}}\left[\left(\mu+\frac{\mu_{t}}{\sigma_{k}}\right) \frac{\partial k}{\partial x_{j}}\right]+G_{k}+G_{b}-\rho \varepsilon \\
& -Y_{M}+S_{k}, \\
\frac{\partial(\rho \varepsilon)}{\partial t}+\frac{\partial\left(\rho \varepsilon u_{i}\right)}{\partial x_{i}}= & \frac{\partial}{\partial x_{j}}\left[\left(\mu+\frac{\mu_{t}}{\sigma_{\varepsilon}}\right) \frac{\partial \varepsilon}{\partial x_{j}}\right] \\
& +G_{1 \varepsilon} \frac{\varepsilon}{k}\left(G_{k}+G_{3 \varepsilon} G_{b}\right)-G_{2 \varepsilon} \rho \frac{\varepsilon^{2}}{k}+S_{\varepsilon},
\end{aligned}
$$

where

$$
\left\{\begin{array}{l}
G_{k}=\mu_{t}\left(\frac{\partial u_{i}}{\partial x_{j}}+\frac{\partial u_{j}}{\partial x_{i}}\right) \frac{\partial u_{i}}{\partial x_{j}} \\
G_{b}=\beta g_{i} \frac{\mu_{t}}{\operatorname{Pr}_{t}} \frac{\partial T}{\partial x_{i}} \\
\beta=-\frac{1}{\rho} \frac{\partial \rho}{\partial T} \\
Y_{M}=2 \rho \varepsilon M_{t}^{2} \\
M_{t}=\sqrt{\frac{k}{a^{2}}} \\
a=\sqrt{\gamma R T}
\end{array}\right.
$$

where $G_{k}$ is the turbulent kinetic energy generated by the average velocity gradient, $G_{b}$ is generated by the turbulent 


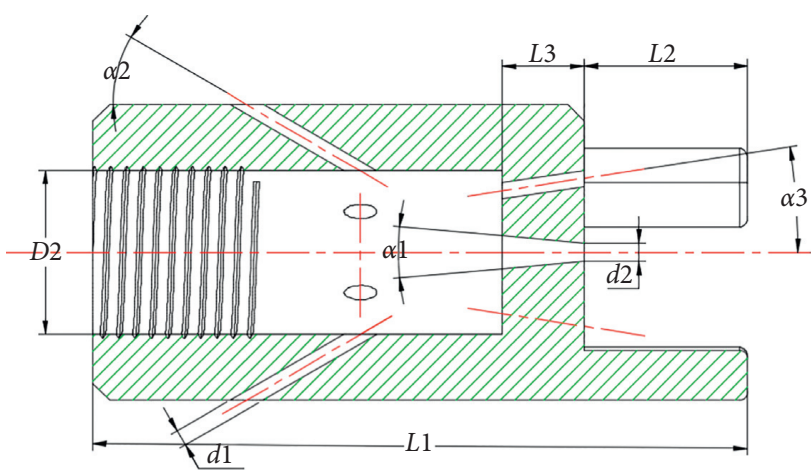

(a)

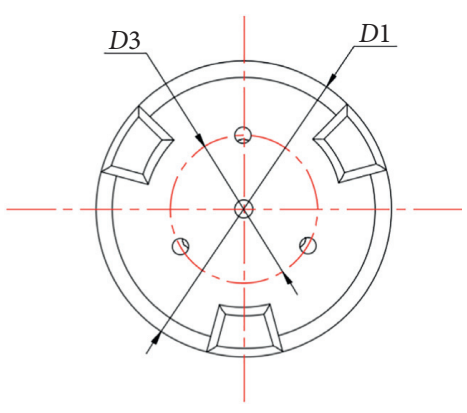

(b)

FIGURE 1: Structure diagram of the self-propelled multihole jet bit with the support plate.

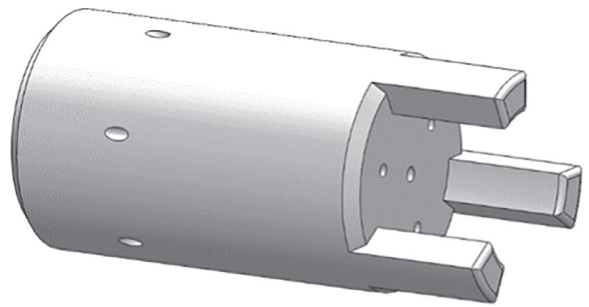

Figure 2: Geometric model.

kinetic energy caused by buoyancy, and $Y_{M}$ is the influence of compressible turbulence pulsation expansion on the total dissipation rate. $C_{1 \varepsilon}, C_{2 \varepsilon}$, and $C_{3 \varepsilon}$ are the empirical constants of the standard $\mathrm{k}-\varepsilon$ turbulence model, and the default values in FLUENT are $C_{1 \varepsilon}=1.44, C_{2 \varepsilon}=1.92$, and $C_{3 \varepsilon}=0.09$. The readings for $\sigma_{k}$ and $\sigma_{\varepsilon}$ are the Puente numbers corresponding to the turbulence kinetic energy and turbulence dissipation rate, respectively. The default values for FLUENT are $\sigma_{k}=1.0$ and $\sigma_{\varepsilon}=1.3 . \mathrm{Pr}_{t}$ is the Prandtl number of turbulence, and $\mathrm{Pr} t=0.85$ by default. $\mathrm{g}_{i}$ is the component of gravitational acceleration in the direction of $i ; \beta$ is the coefficient of thermal expansion; $M_{t}$ is the turbulent Mach number; and $a$ is the speed of sound.

3.2. Meshing. As shown in Figure 3, it is a schematic diagram of mesh division of the three-dimensional model of the fluid domain. This model adopts the nonuniform mesh size, so as to capture the small features in the model. The left part of the grid model is the fluid inlet boundary, and the red part is the fluid outlet boundary. The mesh is much finer when the flow field gradient is large, such as the nozzle outlet and the bottom wall. MESHING module in ANSYS has the function of grid quality inspection. The highest, lowest, and average mass index of the grid divided by this model is 0.99955 , 0.38299 , and 0.87799 . Generally speaking, if the mesh quality is higher than 0.8 , it is considered as good mesh and can complete simulation tasks with high quality.

3.3. Boundary Conditions. Inlet boundary: the inlet boundary of absolute velocity is adopted, the velocity

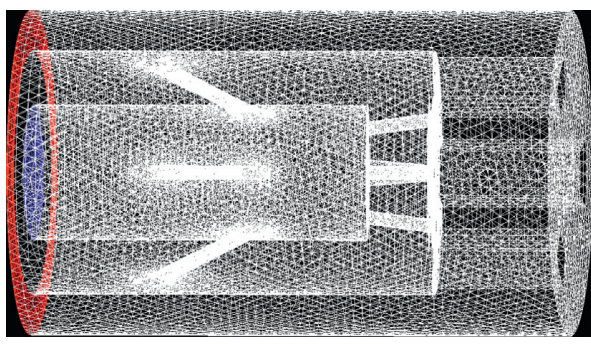

FIgURE 3: Grid division of the computing domain.

direction is the normal direction of the inlet, the absolute velocity of the inlet is $15 \mathrm{~m} / \mathrm{s}$, and the radial velocity is $0 \mathrm{~m} / \mathrm{s}$.

Outlet boundary: both forward and backward outlet pressure adopt environmental pressure. Static pressure $P_{\mathrm{s}}=0.2 \mathrm{MPa}$.

Wall condition: the solid wall boundary without slip adiabatic is adopted, and the particles will bounce back into the calculation domain after hitting the wall.

3.4. Material Setting. The simulated jet medium was water solution of pure water and polyacrylamide (PAM), and the material of the pure water jet was set as $1 \mathrm{~g} / \mathrm{cm}^{3}$ with a viscosity of $0.85 \mathrm{~Pa}$.s. The polymer additive jet has a customized fluid density of $1.5 \mathrm{~g} / \mathrm{cm}^{3}$ and viscosity of $0.39 \mathrm{~Pa} \cdot \mathrm{s}$ [35]. In this paper, when comparing the flow fields of the two jets, only the material settings are different, and the others all use the same settings.

\section{Numerical Simulation Calculation Results and Analysis}

4.1. Comparison of Flow Field Characteristics between Pure Water Jet and Polymer Additive Jet. The flow profile of the pure water jet and the polymer additive jet in the cross section of the bit center axis is shown in Figure 4. On the left is the velocity distribution cloud diagram of the polymer additive jet, and on the right is the velocity distribution cloud diagram of the pure water jet. The two fluids are ejected at high speed through the forward and backward holes of the drill bit, wherein the fluid ejected through the forward hole mainly breaks the rock. The effect of the fluid ejected 
Velocity $(\mathrm{m} / \mathrm{s})$

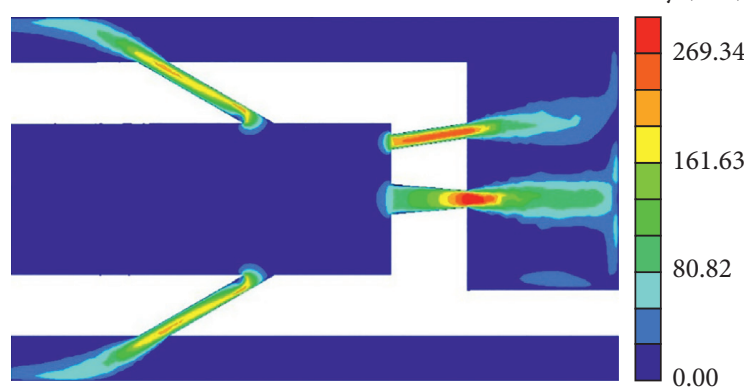

(a)

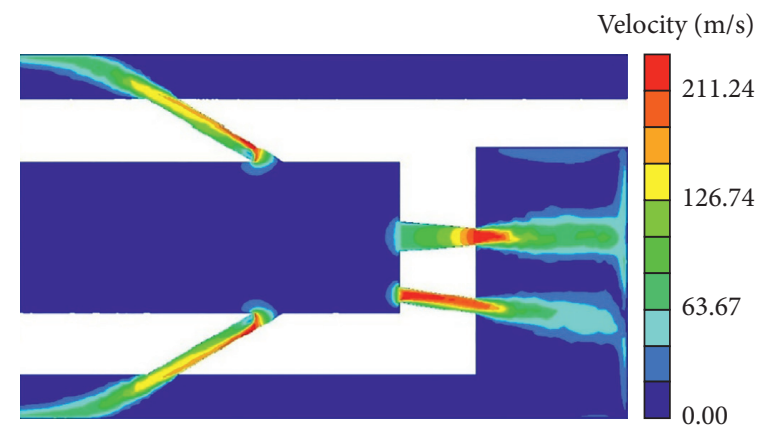

(b)

FIGURE 4: Velocity cloud diagram of cross section of two kinds of fluid in the center axis of the jet bit.

through the rear hole is mainly to ream the hole and provide the self-feeding force of the drill bit. Under the same conditions, the two jet decay laws are similar, and the difference is the polymer additive jet at the nozzle exit speed is much larger. The reason is that the interaction between the pure water jet and the surrounding stationary fluid is larger and the velocity attenuation is serious.

Therefore, under the same pump pressure, polymer additive jet has better rock-breaking effect than the water jet. That is to say, adding polymer to water can improve the efficiency of rock breaking.

4.2. Velocity Distribution of the Forward Jet. Figure 5 shows the cloud diagram of velocity distribution of the polymer additive jet in the forward jet of the drill bit. The section shown in the figure includes a circular hole, a central hole, and a support plate. The cone angle design of the central hole forms a smaller jet diffusion angle, which makes the jet more dense and the more concentrated the energy. It can be clearly seen from the figure that the central hole designed by cone angle has longer jet isokinetic core than other circular common holes and has greater impact force and better rockbreaking effect than other circumferential hole jets. Therefore, the forward central hole plays a major role in rock breaking. Since the other three holes with the same circular distribution are at a certain angle with the central axis of the drill bit, the jet flow formed by the three holes with the same circular distribution will have a certain shear force when hitting the rock, and such shear force is also conducive to rock breaking.

4.3. Velocity Distribution of the Backward Jet. The flow pattern of the backward orifice jet velocity distribution of the self-propelled porous jet drill bit is shown in Figure 6. There are a total of six backward orifices, which are evenly distributed on the same circumference. Part of the fluid enters into the nozzle and jets to the annulus from the inside of the drill bit, forming a backward jet flow. The maximum velocity of the fluid at the outlet of the backward orifice reaches $203.2 \mathrm{~m} / \mathrm{s}$. Under the influence of the surrounding fluid confining pressure, the velocity of the backward jet dissipates somewhat. When the jet finally impacts the rock, the impact
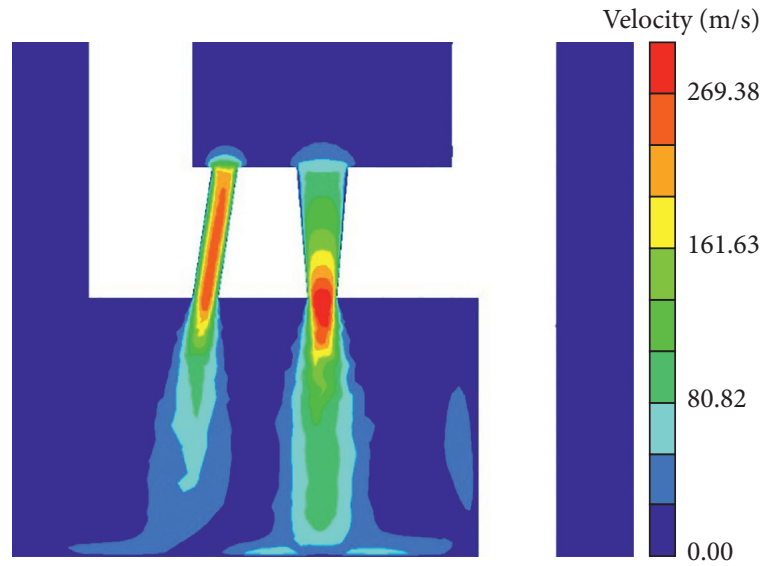

FIGURE 5: Forward jet velocity cloud diagram of the jet bit.

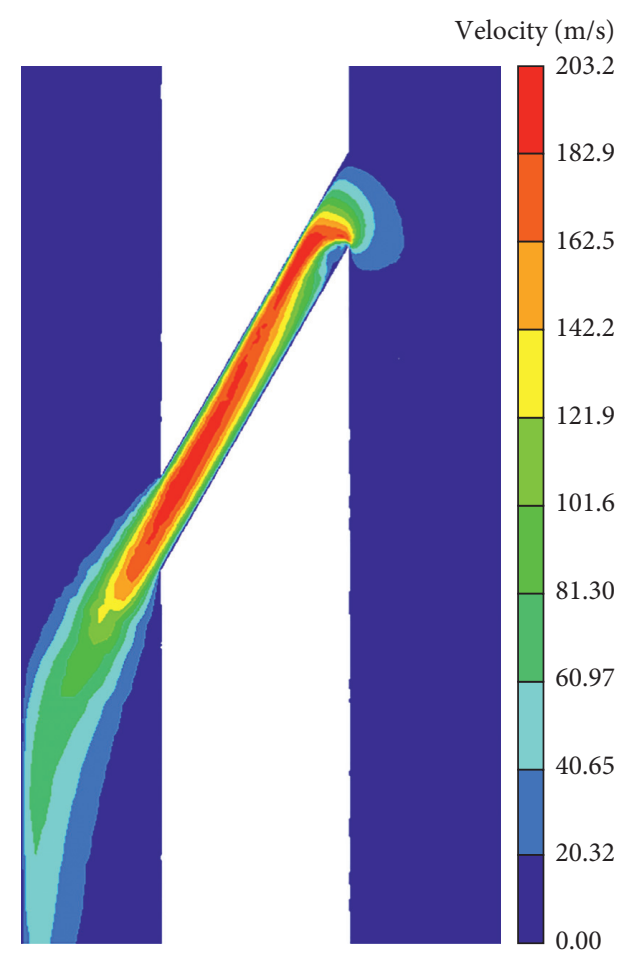

FIGURE 6: Backward jet velocity cloud diagram of the jet bit. 
velocity is still as high as $101.6 \mathrm{~m} / \mathrm{s}$, and the high-speed fluid generates strong shear stress on the wall of the hole, making it more conducive to expanding the aperture of the broken rock.

In addition, the strong propulsive force generated by the backward jet of the polymer additive forms the main driving force for the bit to realize self-propulsion. Due to the viscosity of the polymer additive solution, the cuttings generated at the bottom of the well are more easily discharged through the backward jet.

\subsection{Downhole Flow Field Analysis}

4.4.1. Velocity Distribution. As shown in Figure 7, after the jet of the polymer additive impinges on the bottom hole, the jet fluid flows radially along the bottom hole under the restriction of the bottom hole, forming a diffuse flow with high speed along the bottom hole. This layer of diffuse flow from the polymer additive solution drives the cuttings at the bottom of the well to move laterally together to achieve timely cutting removal.

The research shows that when the wellbore boundary condition is no slip wall surface, the maximum diffuse flow velocity appears within the height range of $0.5 \mathrm{~mm}$ from the wellbore bottom, and the maximum diffuse flow velocity value can reach $50 \%$ to $80 \%$ of the exit velocity of the jet nozzle. A section, $0.5 \mathrm{~mm}$, from the bottom of the well is taken as the research object, and the velocity distribution cloud diagram of the flow field in this section is shown in Figure 8. It can be seen from the figure that four circular high-speed regions are distributed in the velocity distribution cloud map, which is the cross section of the jet formed by the forward jet. In addition, the flow velocity of the jet formed by the forward central nozzle at the bottom of the well is obviously greater, and the crushing depth will also be deeper. The three white areas in the figure are the zero velocity areas formed by the support plate. The staggered arrangement with the other three nozzles with the same circular distribution is to reduce the influence of the support plate on the jet flow. Moreover, the fluid flow velocity around the circular arc surface of the support plate is high, and the crushed rock debris does not accumulate on the inner circular arc surface of the support plate, so as to timely clean the bottom rock cuttings.

Figure 9 shows the cloud diagram of the velocity distribution at the bottom of the well of the pure water jet. It can be seen from the figure that the velocity distribution at the bottom of the well of the pure water jet is roughly the same as that of the polymer additive jet, but the corresponding velocity is much smaller. The maximum impact velocity of the pure water jet hitting the bottom of the well is about $95 \mathrm{~m} / \mathrm{s}$, but the maximum impact velocity of the jet with polymer added can reach $106 \mathrm{~m} / \mathrm{s}$. This proves that the polymer additive jet has better rock-breaking effect.

4.4.2. Pressure Distribution. Figure 10 shows the cloud diagram of static pressure distribution at the bottom of the well of the polymer additive jet. Compared with other three holes

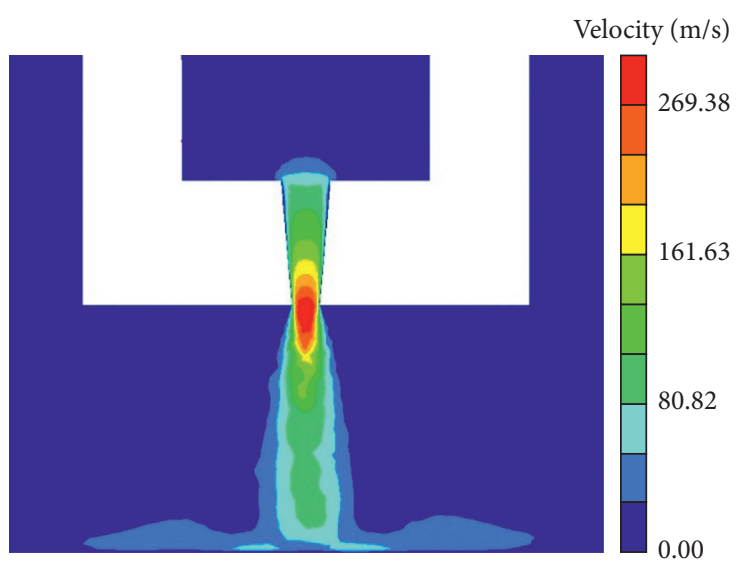

Figure 7: Backward jet velocity cloud diagram of the jet bit.

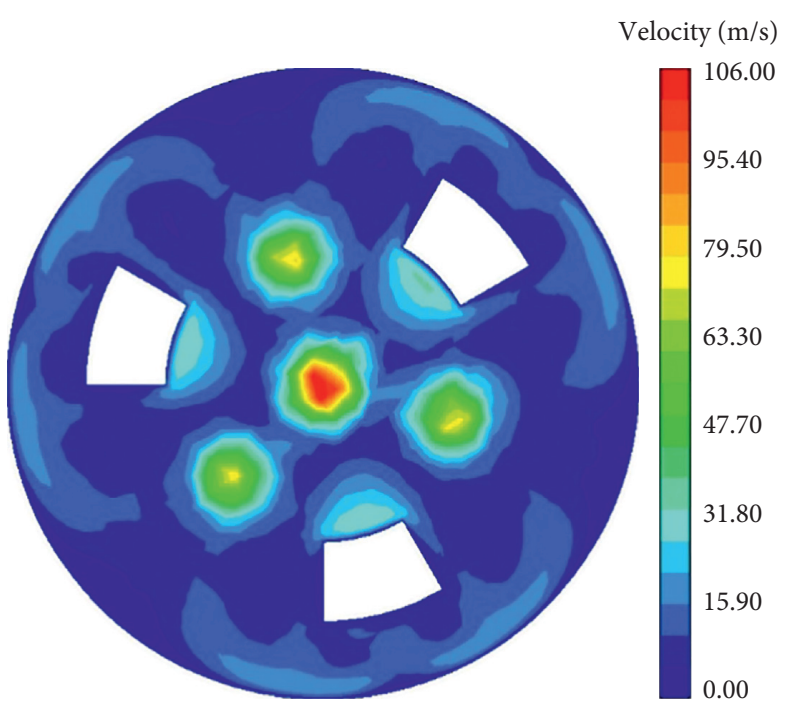

FIGURE 8: Bottom hole velocity distribution cloud image of the polymer additive jet.

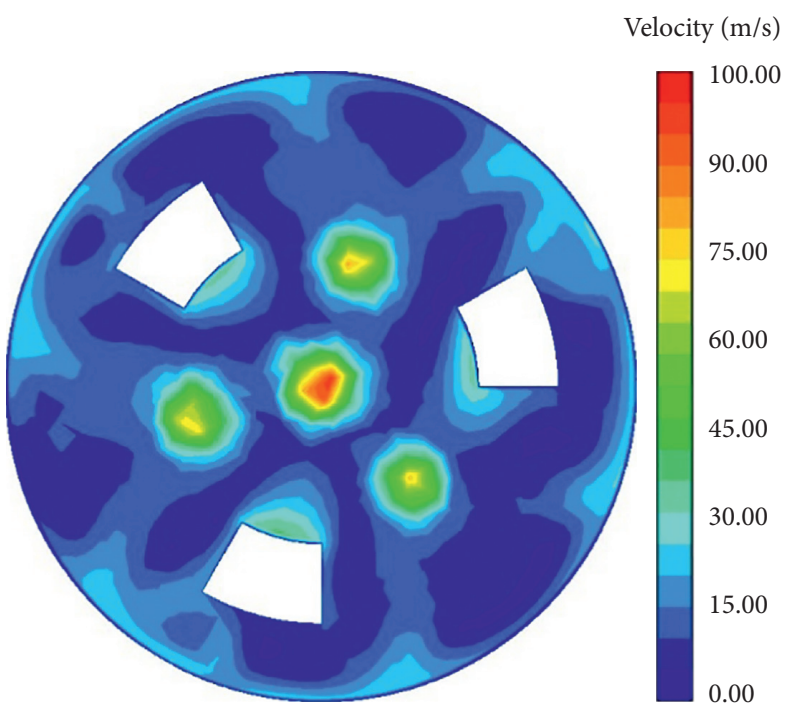

FIGURE 9: Bottom hole velocity distribution cloud image of the pure water jet. 


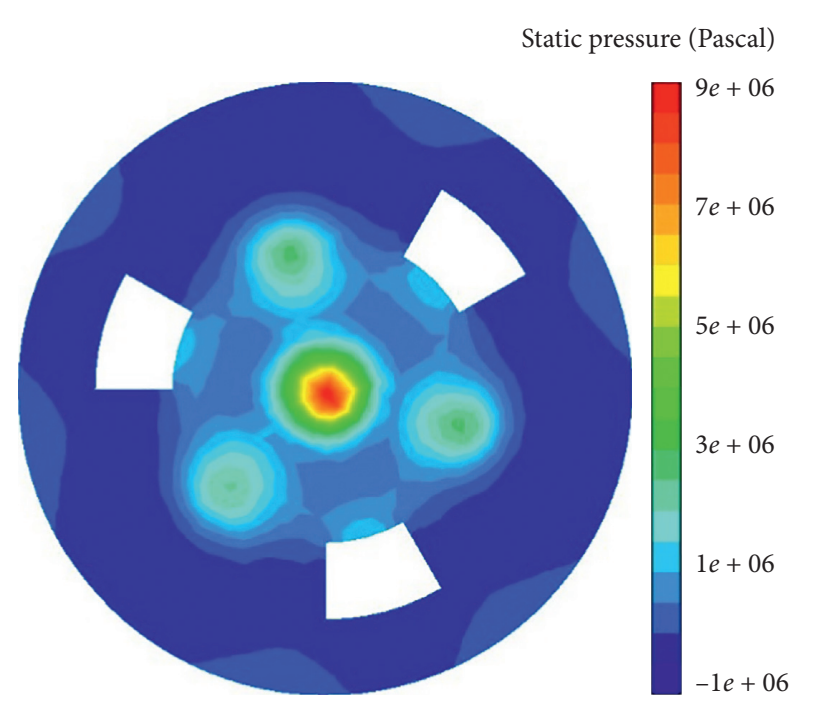

FIGURE 10: Bottom hole static pressure distribution cloud image of the polymer additive jet.

with the same circular distribution, the static pressure of the jet formed by the forward central hole is much larger. The jet static pressure formed by the central hole is at most about $9 \mathrm{MPa}$, while the jet static pressure formed by the holes with the same circular distribution is at most about $4 \mathrm{MPa}$. And all the static pressure areas formed showed a decreasing trend from the center to the surrounding area.

Figure 11 shows the cloud diagram of the bottom hole dynamic pressure distribution of the jet flow of the polymer additive. The dynamic pressure distribution is quite different from the static pressure distribution. The area where the jet impinges on the bottom of the well is the area with the minimum dynamic pressure, and the surrounding dynamic pressure gradually increases. When it is close to the wall of the well, the dynamic pressure gradually decreases.

\section{Experimental}

5.1. Material. The polymer used in the experiment is polyacrylamide (PAM), which is a water-soluble linear polymer synthesized with a high degree of polymerization. PAM density is $1.32 \mathrm{~g} / \mathrm{cm}^{3}$. Because PAM has stronger shear resistance than other polymers and is easily soluble in water, it is particularly suitable for fluid conveying systems with high shear local resistance elements. The molecular weight of PAM used in this experiment is over 10 million, bought in China Xinqi chemical plant, located in Heluo Town, Gongyi City, Henan Province.

5.2. Sample Preparation. Wang et al. [5-7] showed that, with the increase of additive concentration, the crushing depth of the rock first increased and then decreased, and there was an optimal concentration of the polymer additive, so the concentration of the additive was selected as $200 \mathrm{mg} / \mathrm{L}$ in this experiment. When preparing polymer additive aqueous solution, first $5000 \mathrm{mg} / \mathrm{L}$ high concentration aqueous solution is prepared, and then $200 \mathrm{mg} / \mathrm{L}$ required concentration

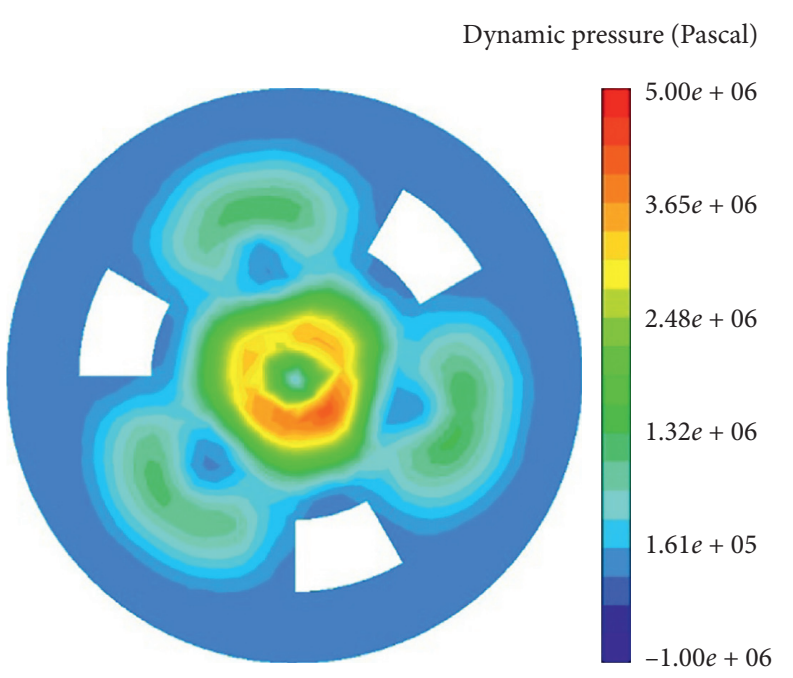

FIGURE 11: Bottom hole dynamic pressure distribution cloud image of the polymer additive jet.

polymer additive solution is prepared, it is fully stirred ( $24 \mathrm{~h}$ ) to make the additive mix evenly, and uniform jet properties are obtained, so as to ensure the maximum performance of the additive. Because the polymer chain of polyacrylamide in the polymer additive solution is easy to break during the impact process, in order to ensure the comparability of the experiment, the additive solution is used once in this experiment instead of repeated use.

5.3. Experimental Equipment. This experiment was carried out in Southwest Petroleum University, which mainly studied and compared the rock-breaking effect of the pure water jet and polymer additive jet. The experimental device is shown in Figure 12, including multistage pump, pipeline, throttle valve, water tank, pressure gauge, electronic flowmeter, water jet nozzle, and jet rock-breaking system. The jetting rock-breaking system can adjust the jetting distance freely. The highest pressure of the multistage pump can reach $40 \mathrm{MPa}$, and the volume of the water tank is $20 \mathrm{~L}$. The rocks used in the experiment are natural sandstone. The experimental nozzle adopts a straight single-hole nozzle with a cone angle of $12.3^{\circ}$, with a nozzle outlet diameter of $4 \mathrm{~mm}$, and with an outlet length of $10 \mathrm{~mm}$.

5.4. Condition Setting. The two groups of experiments adopted the same injection pressure of $30 \mathrm{MPa}$, the same injection time of $60 \mathrm{~s}$, and the same rock to be broken, with only difference in drilling fluid and spraying distance. The rock used in the experiment is natural sandstone, and the crushing effect is characterized by the volume of rock breaking by fixed time and fixed-point impact crushing. In order to make the experimental data accurate and reliable and reduce the influence of experimental error, the polymer additive jet experiment and pure water jet experiment were repeated three times, and the average value of the results of the three experiments was taken as the rock-breaking volume. 


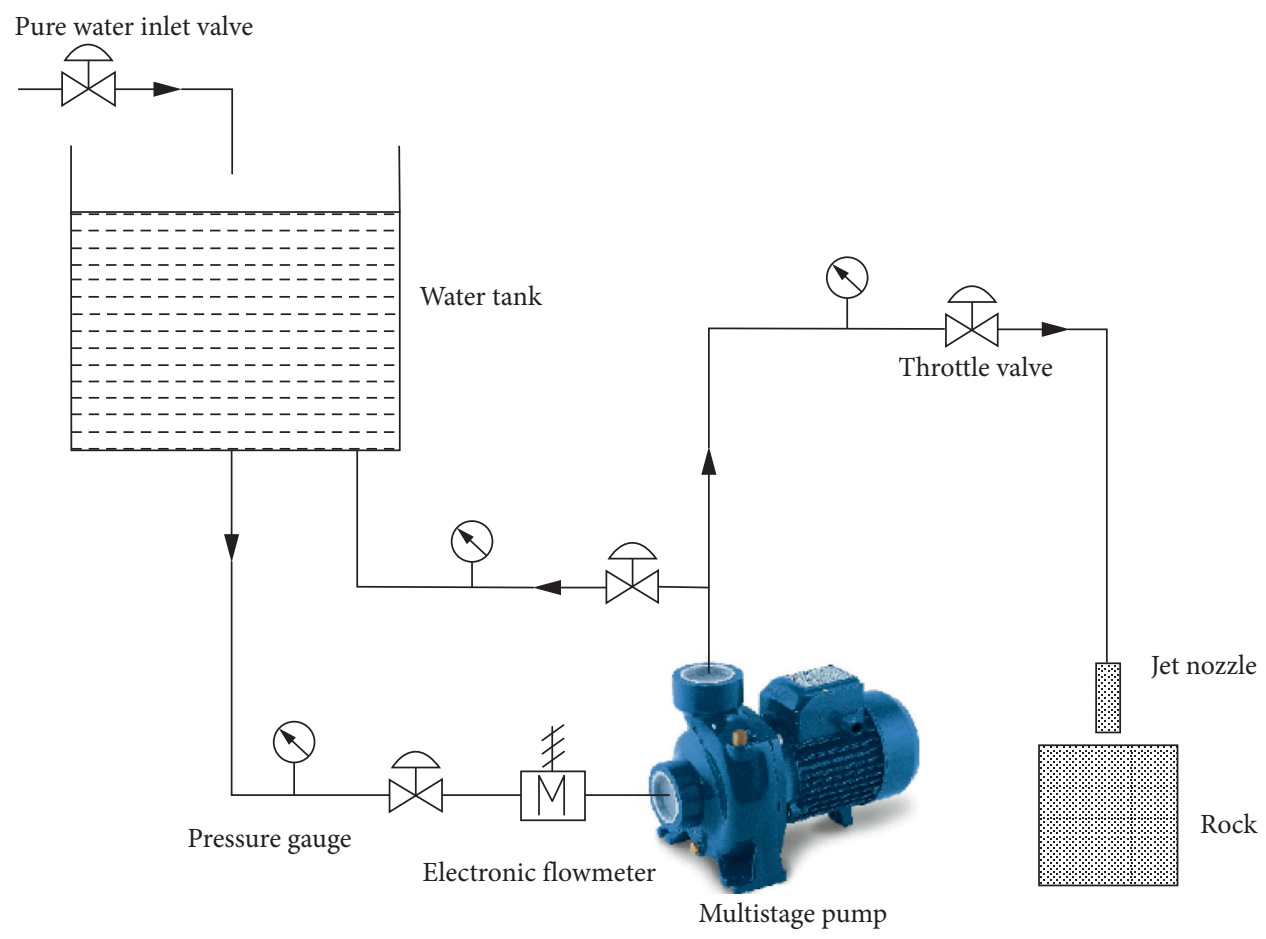

FIGURE 12: Schematic diagram of experimental equipment.

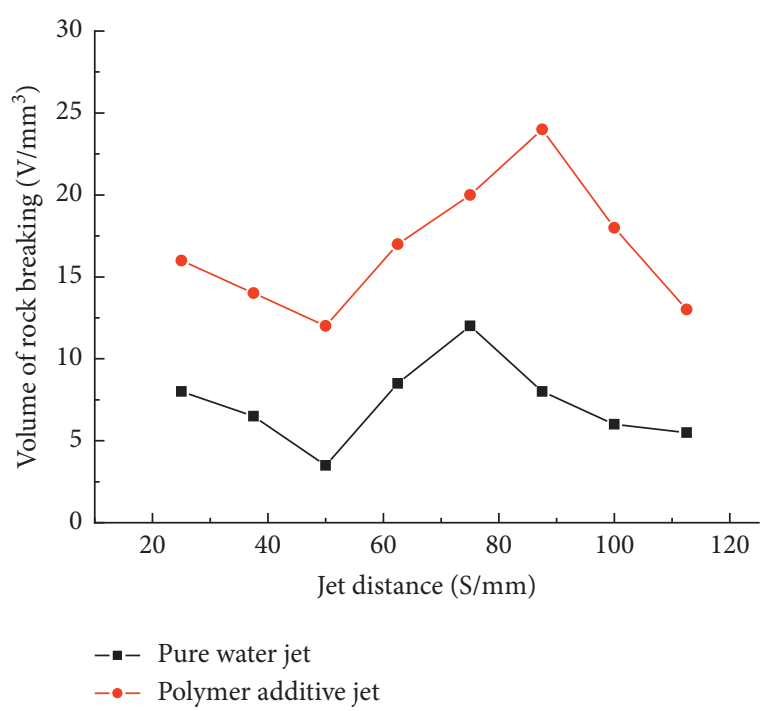

FIGURE 13: Comparison of rock-breaking effect between water jet and polymer additive jet.

5.5. Experimental Results. The experimental results are shown in Figure 13, which are the comparison curve of rock-breaking volume between the additive jet and water jet. It can be seen from the diagram that the rock-breaking effect of the pure water jet and polymer additive jet shows the trend of first decreasing and then increasing with the increase of spray distance. When the optimal spray distance is reached, the rock-breaking effect decreases with the increase of spray distance. In this experiment, the optimal spray distance of the pure water jet is $75.2 \mathrm{~mm}$, while the optimal spray distance of the polymer additive is about
$87.5 \mathrm{~mm}$, and the optimal spray distance of the polymer additive jet increases $12.3 \mathrm{~mm}$ compared with that of the pure water jet. This indicates that the polymer additive increases the rock-breaking effect of the jet.

Before the spray distance of the two jets reaches the optimal spray distance, the trend of rock-breaking effect of the two jets varies with the spray distance which is approximately the same. However, when the spray distance of the two jets reached the optimal spray distance, the rockbreaking efficiency of the polymer additive jet decreased faster with the spray distance than that of the pure water jet.

Under the same conditions, the rock-breaking volume of the additive jet is about twice or more than that of the pure water jet. Moreover, the optimal spray distance of the additive jet is larger than that of the pure water jet. This is because the viscosity of the jet medium increases with the addition of polymer additives, which changes the structural characteristics of the jet. The axial velocity attenuation of the jet becomes slower, and the effective jetting distance of the jet increases. It shows that the polymer additive jet has more impact force and better rock-breaking effect. The experimental results are in good agreement with the numerical simulation results, indicating that the numerical simulation method is reliable in analyzing and designing the flow field characteristics of the jet bit.

\section{Conclusions}

Based on the above study, we can draw the following conclusions:

(1) In this paper, a self-propelled multihole jet bit with the support plate is designed, which can prevent the 
drill bit from jamming due to the jet nozzle against the bottom of the well during the drilling process

(2) When the jet fluid uses the polymer additive solution, the rock-breaking volume of the polymer additive jet is about twice or more than that of the pure water jet, and its optimal jet distance will increase accordingly, which indicates that the polymer additive jet has more efficient rock-breaking efficiency

(3) It can be known from the bottom hole velocity distribution cloud chart and bottom hole pressure distribution cloud chart setup by polymer additive that the forward central nozzle designed by cone angle has more efficient rock-breaking efficiency and longer isovelocity core than other common nozzles with uniform distribution in circumference

\section{Data Availability}

All data included in this study are available upon request by contact with the corresponding author.

\section{Conflicts of Interest}

The authors declare that there are no conflicts of interest regarding the publication of this paper.

\section{Acknowledgments}

This research was funded by State Key Laboratory of Petroleum Resources and Prospecting, China University of Petroleum, Beijing, grant number PRP/open-1610 and the National Natural Science Foundation of China, grant number 51804267.

\section{References}

[1] R. S. Rosler and S. G. Bankoff, "Large-scale turbulence characteristics of a submerged water jet," AIChE Journal, vol. 9, no. 5, pp. 672-676, 1963.

[2] G. S. Li, C. Yi, J. L. Niu et al., "Experimental study on high polymer additive (super water) jet for rock erosion efficiency," Oil Drilling Technology, vol. 29, no. 6, Article ID 1001-0890, 2001.

[3] C. W. Liu and Z. M. Li, "Using LDV to study the flow field of polymer additive jets," in Proceedings of the 1999 National Hydrodynamics Conference, pp. 123-128, Beijing, China, 1999.

[4] Z. M. Li and C. W. Liu, "Experimental study on jet structure characteristics of polymer additive," Drilling Fluid and Completion Fluid, vol. 6, pp. 1-3, 1999.

[5] S. J. Wang, R. H. Wang, and Y. H. Bu, "Laboratory study on drilling by high molecular weight additive jetting," Oil Driling \& Production Technology, vol. 21, no. 1, pp. 21-24, 1999.

[6] R. H. Wang, S. J. Wang, and Y. H. Bu, "Polymer additive jet structure characteristics," Journal of China University of Petroleum (Edition of Natural Science), vol. 22, no. 1, pp. 38-40, 1998.

[7] S. J. Wang, R. H. Wang, and W. D. Zhou, "Influences of macromolecular additives on rotary-jet configuration characteristics," Petroleum Drilling Techniques, vol. 26, no. 3, pp. 55-62, 1998.
[8] Y. Y. Yang, Z. H. Shen, R. H. Wang et al., "Velocity profile of abrasive suspension swirling jet and influence of additive polyacrylamide," Journal of China University of Petroleum (Edition of Natural Science), vol. 29, no. 1, pp. 34-36, 2005.

[9] Y. Y. Yang, R. H. Wang, Z. H. Shen et al., "Experimental study on velocity distribution of swirling jet with polyacry-lamide fluid," Journal of China University of Petroleum (Edition of Natural Science), vol. 25, no. 6, pp. 38-41, 2001.

[10] Y. Y. Yang and G. S. Li, "Experimental study on ultra-high pressure abrasive jet breaking and cutting," Petroleum Drilling Techniques, vol. 3, no. 3, pp. 4-5, 2002.

[11] Y. Y. Yang, R. H. Wang, and W. D. Zhou, "Study on the effect of PAM on abrasive jet flow," Petroleum Drilling Techniques, vol. 29, no. 1, pp. 4-6, 2001.

[12] D. Feng, L. Shi, C. Guo, F. Wang, and Y. Chen, "Numerical and experimental study on the flow characteristics of abrasive slurry jet with polymer additives," The International Journal of Advanced Manufacturing Technology, vol. 95, no. 9-12, pp. 3289-3299, 2017.

[13] G. Seifert, R. Boing, X. L. Wu et al., "Additives are used to improve the effect of high pressure water jet in tunneling," Colliery Mechanical \& Electrical Technology, vol. 5, no. 12, pp. 53-63, 1982.

[14] X. P. Jiang, S. G. Hu, and S. Y. Zhong, “An experimental study of abrasive water jet and improvement on the performance with high polymer drag reduction," Journal of University of Shanghai for Science and Technology, vol. 15, no. 2, pp. 87-91, 1993.

[15] H. N. Zuo, Experimental Study on Adding Polymers in Abrasive Water Jet, Diss, Hunan University of Technology, Xiangtan, China, 2013.

[16] J. H. Pei and Z. W. Bai, "Influence on cutting quality generated by high polymeric additives abrasive water jet," Machine Tool \& Hydraulics, vol. 44, no. 15, pp. 142-146, 2016.

[17] J. H. Pei and D. Hu, "Experimental study on cutting taper by high polymeric additives abrasive water jet," Manufacturing Technology \& Machine Tool, vol. 5, no. 34, pp. 83-86, 2016.

[18] J. H. Pei, Y. L. Wang, and J. H. Chen, "Theory and experiment to influences of macromolecular additives on jet cutting," Journal of Lanzhou Petrochemical College of Technology, vol. 16, no. 1, pp. 4-6, 2016.

[19] Y. Y. Zhang, X. C. Wang, and D. Hu, "Influence of adding a small amount high molecular polymer on cutting performance of abrasive water jet," Journal of Sichuan University (Engineering Science Edition), vol. 46, no. 5, pp. 181-187, 2014.

[20] W. Dickinson and R. W. Dickinson, "Horizontal radial drilling system," in Proceedings of the SPE California Regional Meeting, Society of Petroleum Engineers, Bakersfield, CA, USA, March 1985.

[21] H. Y. Li, C. J. Wang, L. H. Shi et al., "Application and development of drilling and completion of the ultrashort-radius radial well by high pressure jet flow techniques," in Proceedings of the International Oil and Gas Conference and Exhibition in China, Beijing, China, November 2000.

[22] W. Dickinson, H. Dykstra, R. Nordlund et al., "Coiled-tubing radials placed by water-jet drilling: field results, theory, and practice," in Proceedings of the SPE Annual Technical Conference and Exhibition, SPE, Houston, TX, USA, October 1993.

[23] C. W. Landers, "Method of and apparatus for horizontal well drilling," US Patent 6125949A, 2000.

[24] B. M. Henry, "Horizontal directional drilling in wells," US Patent 6889781B2, 2005. 
[25] X. H. Zhu and W. Chen, "Analysis of influencing factors and parameter optimization of self-propelling force of self-propelling nozzle," Research and Progress in Hydrodynamics, vol. 33, no. 1, pp. 89-97, 2018.

[26] P. Buset, M. Riiber, A. Eek et al., "Jet drilling tool: cost-effective lateral drilling technology for enhanced oil recovery," in Proceedings of the SPE/ICoTA Coiled Tubing Roundtable, Society of Petroleum Engineers, Houston, TX, USA, March 2001.

[27] G. Bi, D. J. Ma, G. S. Li et al., "Hydraulic jet side tracking radial horizontal well extension capacity," Fault Block Oil and Gas Field, vol. 23, no. 5, pp. 643-647, 2016.

[28] G. Bi, G. S. Li, Z. Qu et al., "Rock breaking effect of selfpropelled rotary jet drill," Acta Petrolei Sinica, vol. 37, no. 5, pp. 680-687, 2016.

[29] G. L. Yang, W. H. Zhou, and F. Liu, "Flow field simulation of high-pressure water jet nozzle based on fluent," Journal of Lanzhou University of Technology, vol. 34, no. 2, pp. 49-52, 2008.

[30] G. Z. Wang, C. Y. Ran, B. Deng et al., "Study on jet characteristics of long-distance flushing nozzle based on FLUENT," Hydraulics Pneumatics \& Seals, vol. 36, no. 1, pp. 31-34, 2016.

[31] D. Y. Zhu, D. Y. Liang, P. W. Shi et al., "Study on the influence of ore jet nozzle structure on jet flow field," Mineralogy \& Metallurgy, vol. 25, no. 6, pp. 65-69, 2016.

[32] K. Shen, J. Yang, L. Ma et al., "Research and application of hydraulic jet composite plugging removal technology," Petrochemical Applications, vol. 30, no. 1, pp. 48-35, 2011.

[33] T. G. Chen, Z. C. Guan et al., Drilling Engineering Theory and Technology, China University of Petroleum, Dongying, China, 2006.

[34] H. J. Zhu, Y. H. Lin, and L. H. Xie, FLUENT Fluid Analysis and Simulation Practical Tutorial, People's Posts and Telecommunications, Beijing, China, 2010.

[35] C. H. Li and Y. Y. Zhang, "Numerical simulation of mechanical degradation of polymer solution under the shaft," Value Engineering, vol. 33, no. 49, pp. 124-126, 2016. 